DAMTP/97-96

\title{
Two-Component Reduction of Nahm's Equations and Hyper-elliptic Solutions
}

\author{
Houari Merabet円 \\ Department of Applied Mathematics \& Theoretical Physics \\ University of Cambridge, Cambridge CB3 9EW, England \\ M.Houari@damtp.cam.ac.uk
}

October 1997

\begin{abstract}
We find the general hyper-elliptic solutions to the two-component reduced Nahm equations proposed in [4]. Elliptic solutions are a special case and can appear only for specific values of the monopole charges.
\end{abstract}

\footnotetext{
${ }^{1}$ Research supported by the EPSRC grant GR/K50641
} 


\section{Introduction}

Monopoles are soliton solutions to the $S U(2)$ Yang-Mills-Higgs equations in $3+1$ dimensions [1]. In the limit when the Higgs potential vanishes, static monopoles are solutions to the Bogomolny equations [2]

$$
F_{i j}=\epsilon_{i j k} D_{k} \phi
$$

where $F_{i j}=\partial_{i} A_{j}-\partial_{j} A_{i}-\left[A_{i}, A_{j}\right]$ and $D_{k} \phi=\partial_{k} \phi-\left[A_{k}, \phi\right]$. The Higgs field $\phi$ and the gauge fields $A_{i}$ are in the adjoint representation of $\mathrm{SU}(2)$. It has been proven long time ago by Nahm [3], that an $N$ charged monopole solutions of the Bogomolny equations are uniquely related to the corresponding solutions of Nahm's equations

$$
\frac{d T_{k}(s)}{d s}=\frac{1}{2} \epsilon_{i j k}\left[T_{i}(s), T_{j}(s)\right]
$$

where the $N \times N$ matrices $T_{i}$ are meromorphic functions defined on the interval [0,2], regular on $(0,2)$ with simple poles at $s=0,2$. The residues at each pole form an irreducible $N$ dimensional representation of $s u(2)$. Furthermore, the $T_{i}$ satisfy a reality condition $T_{i}(s)=$

$-T_{i}^{\dagger}(s)=T_{i}^{\dagger}(2-s)$. To each solution of Nahm's equation one can associate a spectral curve defined by

$$
P(\eta, \zeta) \equiv \operatorname{det}\left(\eta+i\left(T_{1}+i T_{2}\right)-2 i T_{3} \zeta-i\left(T_{1}-i T_{2}\right) \zeta^{2}\right)=0
$$

The coefficients of the polynomial (3) are independent of $s$ and arise as constant of integrations of Nahm's equations.

Eqs.(2) are non-linear first order differential equations. Although they are more simpler mathematically than eqs.(1) but still only few solutions are known. In the present paper, we will show how to obtain general solutions for the two-component reduced version of eqs.(2) and discover that all the known solutions appear as special cases. 


\section{Solutions of 2-component reduced Nahm's equations}

Let us recall how one obtains two-component reduced Nahm's equations 四. First, one expresses the Nahm matrices as

$$
T_{i}(s)=x(s) \rho_{i}+y(s) S_{i}
$$

The real functions $x(s)$ and $y(s)$ have to be regular on $(0,2)$ with simple poles at $s=0,2$. The $\rho_{i}$ form an $N$-dimensional representation of so(3), i.e., $\left[\rho_{i}, \rho_{j}\right]=2 \epsilon_{i j k} \rho_{k}$. Whereas the $S_{i}$ are the components of a G-invariant vector in $S^{2 k} V$. Here $G$ is a finite subgroup of $S O(3)$. The $T_{i}$ belong to a two-dimensional space generated by the vetors $\rho_{i}$ and $S_{i}$. Therefore

$$
\begin{gathered}
{\left[S_{i}, \rho_{j}\right]+\left[\rho_{i}, S_{j}\right]=\epsilon_{i j k}\left(\alpha \rho_{k}+\beta S_{k}\right)} \\
{\left[S_{i}, S_{j}\right]=\epsilon_{i j k}\left(\gamma \rho_{k}+\delta S_{k}\right) .}
\end{gathered}
$$

Nahm's equations reduce then to

$$
\frac{d x}{d s}=2 x^{2}+\alpha x y+\gamma y^{2} ; \quad \frac{d y}{d s}=\beta x y+\delta y^{2}
$$

Eqs.(7) represent a two-dimensional system of coupled non-linear differential equations. One can arrive at the same system by considering Nahm's matrices as having values on the root space of simple Lie algebras [6, 7]

$$
T_{i}(s)=\sum_{r \in R} e_{r, i}(s) E_{r}
$$

where $R$ is a set of roots and $\mathbf{e}_{r}=\left(e_{r, 1}, e_{r, 2}, e_{r, 3}\right)$ are three-dimensional vector fields. The matrices $E_{r}$ are the non-commuting generators of a simple Lie algebra. The reality condition for the Nahm matrices $T_{i}$ implies that $\mathbf{e}_{-r}(s)=-\mathbf{e}_{r}^{*}(s)$ while eqs.(2) become

$$
\frac{d \mathbf{e}_{r}(s)}{d s}=\frac{1}{2} \sum_{w \in R} N_{w, r-w} \mathbf{e}_{w}(s) \wedge \mathbf{e}_{r-w}(s)
$$

with constraints

$$
\sum_{r \in R} r_{\mu} \mathbf{e}_{r}(s) \wedge \mathbf{e}_{-r}(s)=0, \quad \mu=1, \ldots, n
$$


where the constants $n$ and $N_{r, w}$ are respectively the rank and the structure constants of a simple Lie algebra. The $\left(r_{1}, \ldots, r_{n}\right)$ is the root vector. The two-component reduction (4) reads in terms of the vector fields $\mathbf{e}_{r}$

$$
\mathbf{e}_{r}(s)=x(s) \mathbf{a}_{r}+y(s) \mathbf{b}_{r}
$$

The constant vectors $\mathbf{a}_{r}$ and $\mathbf{b}_{r}$ can be either real or purely imaginary and they satisfy

$$
\begin{gathered}
\frac{1}{2} \sum_{s \in R} N_{s, r-s} \mathbf{a}_{s} \wedge \mathbf{a}_{r-s}=2 \mathbf{a}_{r}, \quad \frac{1}{2} \sum_{s \in R} N_{s, r-s} \mathbf{b}_{s} \wedge \mathbf{b}_{r-s}=\gamma \mathbf{a}_{r}+\delta \mathbf{b}_{r} \\
\sum_{s \in R} N_{s, r-s} \mathbf{a}_{s} \wedge \mathbf{b}_{r-s}=\alpha \mathbf{a}_{r}+\beta \mathbf{b}_{r} .
\end{gathered}
$$

The constraints (10) read in terms of $\mathbf{a}_{r}$ and $\mathbf{b}_{r}$

$$
\sum_{r \in R} r_{\mu}\left(\mathbf{a}_{r} \wedge \mathbf{b}_{-r}-\mathbf{b}_{r} \wedge \mathbf{a}_{-r}\right)=0, \quad \mu=1, \ldots, n
$$

The key idea for solving (7) is to find a constant linear transformation $u=a x+b y$ and $v=c x+d y$ such that

$$
\frac{d u}{d s}=q_{1} u^{2}+q_{2} v^{2}, \quad \frac{d v}{d s}=q_{3} u v
$$

where $q_{1}, q_{2}$ and $q_{3}$ are real constants. It is a simple exercise to prove that eqs.(7) can always be put in the form (14) provided that the constants $\alpha, \beta, \gamma$ and $\delta$ satisfy some conditions. In the case when $\beta \neq 2$ or -4 and $\gamma \neq 0$, one can choose the following transformation

$$
\begin{gathered}
a=(\beta-2)(\alpha(\beta+2)-4 \delta) ; \quad b=-(\beta-2)^{2}(\alpha+2 \delta) \\
c=d=(\beta-2)^{2}(\beta+4)
\end{gathered}
$$

with the constraint

$$
\gamma=\frac{(\beta \delta-2 \alpha)(\alpha(\beta+2)-4 \delta)}{(\beta-2)(\beta+4)^{2}} .
$$

Let $p=2 q_{1} / q_{3}$ and $k=2 q_{2} / q_{3}$ then

$$
\begin{gathered}
p=(2+\beta) / 2 ; \quad k=(2-\beta) / 2 \\
q_{3}=4(\beta-2)(\alpha \beta+\delta(\beta-4))
\end{gathered}
$$


Let $t=q_{3} s / 2$, then the system (14) reduces to

$$
\frac{d u}{d t}=(2-k) u^{2}+k v^{2}, \quad \frac{d v}{d t}=2 u v .
$$

A remarkable feature of this system is that it involves only one constant instead of four in eqs.(17). The next step is to find a constant of motion for eqs. (18) which will helps us to get rid of one of the variables.

Proposition. If $k$ is a positive integer then the polynomial $a_{k}=b_{k} v^{k-2}\left(v^{2}-u^{2}\right)$ of degree $k$ is a constant of integration of eqs.(18), with $b_{k}$ an arbitrary constant.

It is easy to check that $a_{k}$ is a constant of integration of eqs.(18) by taking its derivative with respect to $t$ and then using (18). From (17) we see that the condition for $k$ to be a positive integer is equivalent to the statement that $\beta$ is a negative even integer. Since $a_{k}$ is a constant of integration of Nahm's equations it should also appear as a coefficient in the spectral curve (3). Therefore the degree $k$ of the polynomial $a_{k}$ satisfies $k \leq N$. The integer $k$ has a simple explanation in the context of the root system approach to Nahm's equations [6, 7]. In the case of the $A_{n}$ root systems $k=n+1$.

Using the expression of $a_{k}$ in the above proposition, we express $u$ as a function of $v$

$$
u^{2}=v^{2}\left(1 \pm \frac{c^{k}}{v^{k}}\right)
$$

where $c^{k}=\left|a_{k}\right| /\left|b_{k}\right|$. Using (18), we get a first-order non-linear differential equation for $v$

$$
v^{\prime 2}=4 v^{4}\left(1 \pm \frac{c^{k}}{v^{k}}\right)
$$

where $v^{\prime}=d v / d t$. To solve (20) we introduce a function $w$ given by $v=c w^{\sigma}$, with $\sigma$ an arbitrary constant. With this new variable eq. 20] reads

$$
w^{\prime 2}=4 \frac{c^{2}}{\sigma^{2}}\left(w^{2(\sigma+1)} \pm w^{(2-k) \sigma+2}\right)
$$

Solutions to eq.(21) are of hyper-elliptic and elliptic types. They are summarized in the following theorem 
TheOREM. Hyper-elliptic solutions to eq.(21) exist if and only if

(i) $\sigma=-1$ and $k \geq 5$ or

(ii) $\sigma=-\frac{1}{2}$ and even $k \geq 8$

Elliptic solution of Weierstrass type exist for the following cases only

(i) $k=3$ and $\sigma= \pm 1$

(ii) $k=4$ and $\sigma= \pm \frac{1}{2}$ or \pm 1

(iii) $k=6$ and $\sigma= \pm \frac{1}{2}$.

Proof. For eq.(21) to be an hyper-elliptic differential equation, the right hand side of (21) has to be a polynomial in the variable $w$. In other words, the constants $l=2(\sigma+1)$ and $m=(2-k) \sigma+2$ have to be non-negative integers.

(a) If $l=0$ then $\sigma=-1$ and $m=k$.

The solutions are hyper-elliptic for $k \geq 5$ and elliptic for $k=3,4$.

(b) If $l=1$ then $\sigma=-1 / 2$ and $m=1+k / 2$.

The solutions are hyper-elliptic for any even $k \geq 8$ and elliptic for $k=4,6$.

(c) If $l=2$ then $\sigma=0$ and $m=2$.

The solutions are logarithmics or constants for any $k$.

(d) If $l=3$ then $\sigma=1 / 2$ and $m=3-k / 2$.

The solutions are elliptic for $k=4,6$.

(e) If $l=4$ then $\sigma=1$ and $m=4-k$.

The solutions are elliptic for $k=3,4$.

(f) If $l \geq 5$ and $k \geq 3$ the integer $m$ is negative and there are no hyper-elliptic solutions.

The solutions with $k=3$ and $\sigma= \pm 1$ describe the tetrahedral symmetric 3-monopoles as found in [4], whereas $k=4$ and $\sigma= \pm \frac{1}{2}$ or \pm 1 describe the octahedral symmetric ( 4 and 
5)-monopoles [4, 5]. Finally, the cases $k=6$ and $\sigma= \pm \frac{1}{2}$ are associated with dodecahedral symmetric 7-monopoles [5].

\section{Conclusions}

We have explicitly solved the two-component reduced Nahm equations proposed in 4 . The general solutions are of hyper-elliptic type. Elliptic solutions of Weierstrass type appear only for $k=3,4,6$. The latter correspond to the platonic symmetric monopoles found by [⿶, 包. Hyper-elliptic solutions should describe monopoles with charges $N \geq 7$, but one has to be sure that they satisfy the right boundary conditions, i.e., regular on $(0,2)$ with simple poles at $s=0,2$. The problem then resumes in finding the $N \times N$ matrices $\rho_{i}$ and $S_{i}$ which statisfy the algebra (6). This problem can be simplified if one adopts the root system approach [6]. The problem then consists in solving the systems (12) and (13).

ACKNOWLEDGEMENTS. I am grateful to Tomasz Brzeziński and Nick Manton for their encouragements and valuable suggestions. I wish also to thank Conor Houghton and Sazzad Nasir for their critical reading of this manuscript.

\section{References}

[1] G. t'Hooft, Magnetic monopoles in unified gauge theories, Nucl. Phys. B 79 (1974) 276-284. A.M. Polyakov, Particle spectrum in the quantum field theory, JETP Lett 20 (1974) 194-195.

[2] E. B. Bogomolny, Stability of classical solutions, Sov. J. Nucl. Phys. 24 (1976) 861

[3] W. Nahm, The construction of all self-dual multimonopoles by the ADHM method, in Monopoles in Quantum Field Theory, N.S Craigie, P. Goddard and W. Nahm (eds.), World Scientific, 1982, pp.87-95. See also, N.J. Hitchin, On the construction of monopoles, Commun. Math. Phys. 89 (1983) 145-190. 
[4] N.J. Hitchin, N.S. Manton and M.K. Murray, Symmetric monopoles, Nonlinearity 8 (1995) 661-692.

[5] C.J. Houghton and P.M. Sutcliffe, Octahedral and dodecahedral monopoles, Nonlinearity 9 (1996) 385-401.

[6] T. Brzeziński and H. Merabet, Nahm's equations and root systems, hep-th/9709070, to appear in Czech. J. Phys.

[7] T. Brzeziński and H. Merabet, in preparation. 